\title{
Estudio prospectivo de escenarios de la tecnología en el trabajo en Colombia al $2050^{1}$
}

\section{Prospective study of scenarios of technology at work in colombia at 2050}

DOI: https://doi.org/10.17981/econcuc.40.2.2019.07

Artículo de investigación. Fecha de recepción: 03/04/2019 Fecha de aceptación: 15/08/2019

\author{
Liliana Carolina Rodríguez Valero \\ Universidad Central. (Bogotá, Colombia) \\ lrodriguezv3@ucentral.edu.co
}

Ramón Eduardo Gutiérrez Rodríguez

Universidad Central. (Bogotá, Colombia)

rgutierrezr@ucentral.edu.co

Para citar este artículo:

Rodríguez, L. y Gutiérrez, R. (2019). Estudio prospectivo de escenarios de la tecnología en el trabajo en Colombia al 2050. Económicas CUC, 40(2), 101-116. DOI: http://dx.doi.org/10.17981/econcuc.40.2.2019.07

\section{Resumen}

La innovación y las nuevas tecnologías son uno de los componentes que están transformando los indicadores económicos, sociales y culturales lo cual implica que los mercados laborales implican una reorganización en los mercados laborales se reorganicen. A partir de un estudio prospectivo de tipo mixto esta investigación tiene el propósito de responder a la pregunta ¿cuáles serán los escenarios que tendrá que afrontar la sociedad colombiana en la transformación laboral a partir de las variaciones tecnológicas en el año 2050?. Su utilidad radica en la posibilidad de proponer herramientas para incrementar tareas de investigación, desarrollo e innovación en las universidades, complementando la capacitación de los futuros profesionales, así mismo, desarrollar estrategias de mejoramiento social, con las cuales se incentive el emprendimiento que permita disminuir las tasas de desempleo, producto de los futuros desarrollos tecnológicos. En la metodología, se utiliza el corte cualitativo, se lleva a cabo un panel de expertos y luego, se plantea una matriz de escenarios con base al análisis morfológico de los resultados obtenidos por la aplicación de los instrumentos, entre otros. Se encontró que el gobierno resulta ser un actor clave para influir en el desarrollo de las nuevas tecnologías que se encuentran presentes en la revolución industrial 4.0. Y para concluir con que la sociedad es el pilar de los cambios más significativos y trascendentales. Palabras clave: Prospectiva; tecnología; trabajo; escenarios; innovación

\begin{abstract}
s
Innovation and new technologies are one of the components that are transforming economic, social and cultural indicators, which implies that the labor markets imply a reorganization in the reorganization of labor markets. Based on a prospective study of a mixed type, this research aims to answer the question: what will be the scenarios that Colombian society will have to face in the transformation of employment based on technological variations in 2050? Its usefulness lies in the possibility of proposing tools to increase research, development and innovation tasks in universities, complementing the training of future professionals, as well as developing strategies for social improvement, which will encourage entrepreneurship that reduces unemployment rates as a result of future technological developments. In the methodology, the qualitative cut is used, a panel of experts is carried out and then, a matrix of scenarios is proposed based on the morphological analysis of the results obtained by the application of the instruments, among others. The government was found to be a key actor in influencing the development of new technologies that are present in the 4.0 industrial revolution. And to conclude that society is the pillar of the most significant and farreaching changes.
\end{abstract}

Keywords: Prospective; technology; work; scenarios; innovation

1 Derivado del proyecto de investigación titulado "Estudio prospectivo del futuro del trabajo y la tecnología en Colombia al 2050"2050", desarrollado por la Facultad de Ciencias Administrativas, Económicas y Contables de la Universidad Central durante el segundo semestre de 2017 y el primer semestre de 2018. 


\section{INTRODUCCIÓN}

Esta investigación surgió a partir de un macroproyecto de investigación denominado "Estudio prospectivo del futuro del trabajo y la tecnología en Colombia al 2050", desarrollado por la Facultad de Ciencias Administrativas, Económicas y Contables de la Universidad Central durante el segundo semestre de 2017 y el primer semestre de 2018. En una de las etapas de este proyecto participaron tres estudiantes de la Maestría en Gestión de Organizaciones de la misma Universidad, para llevar a cabo su opción y trabajo de grado de dicha maestría. Como resultado, cada uno de los tres estudiantes realizó un artículo, con el propósito de ser publicado en una revista indexada.

De estos a la fecha, ya se encuentra publicado el artículo "Un estudio prospectivo del trabajo en Colombia al 2050" (Cruz y Gutiérrez, 2018). Si bien la investigación de los candidatos a maestría comparte marcos de referencia teóricos y metodológicos, cada uno aborda un área específica. Así, el trabajo de Cruz y Gutiérrez (2018) aborda los escenarios de trabajo, otro artículo aborda los escenarios de la educación superior y el presente artículo aborda los escenarios de tecnología en el trabajo para Colombia a 2050.

La innovación y las nuevas tecnologías convergen en las transformaciones económicas, ambientales, sociales y culturales que implican una reorganización en los mercados laborales, de bienes y de servicios (Glenn, 2016). Suponiendo todo ello "un progreso material e intelectual del hombre, la creación de condiciones y unidad de la especie, en aras de construir un futuro" (Chumaceiro, Hernández, Meléndez y Acurero, 2017, p. 196). Sin embargo, los cambios proyectados para el 2050 generan preocupación acerca del futuro en aspectos como el mercado laboral, la capacitación, y sobre manera, la inmersión de la innovación y las nuevas tecnologías en la cotidianidad de las sociedades (Quaglia y Plata, 2018).

Por consiguiente, organizaciones internacionales han convergido en desarrollar el Proyecto del Milenio o Millenium Proyect, con el fin de determinar los escenarios positivos y negativos, así como los desafíos que presentará la humanidad para el año 2050, incluyendo entre ellos el desarrollo de la robótica, la Inteligencia Artificial (IA), nanotecnología, la biomedicina y biología sintética, la impresión $3 \mathrm{D}$ y $4 \mathrm{D}$ el cambio medioambiental y demográfico, tecnologías 3.0 y 4.0 (Fundación telefónica, 2018).

La literatura correlacionada representa como el desarrollo tecnológico traerá una profundización de las brechas salariales, implicando un aumento en la concentración de la riqueza, incrementando las tasas de desempleo y la desocupación a nivel mundial, por el desplazo en labores operativas, creando nuevos escenarios y empresas, acorde a las nuevas tendencias (Cordeiro, 2013; Glenn, 2016; Serrano, 2016). Donde la participación gubernamental, en compañía de las organizaciones internacionales, son actores para la creación de estrategias orientadas a contrarrestar los efectos adversos de la revolución 4.0 (Fundación telefónica, 2018; Glenn, 2016; Glenn \& Gordon, 2001; Caro, 2016).

Así, este artículo, es un trabajo de corte investigativo, donde se identifican los desafíos por afrontar para la sociedad colombiana en el ámbito laboral, a raíz, de los cambios tecnológicos esperados para el 2050, de tal forma, que se resuelva la pregunta de investigación: ¿Cuáles serán los escenarios que tendrá que afrontar la sociedad colombiana en la transformación laboral a partir de las variaciones tecnológicas en el año 2050? Con lo cual, se busca aportar a la consolidación de visiones de futuro que se sumen a la base de conocimiento acerca de la prospectiva. 


\section{MARCO TEÓRICO-CONCEPTUAL}

En virtud de los cambios que conlleva una cuarta revolución industrial, se han desarrollado estudios, que plantean escenarios tanto positivos como negativos en los que puede incurrir las sociedades para el año 2050 (Castells, 1999). Convergiendo en que la tecnología desplazará la mano de obra tradicional, pero se crearán nuevos trabajos acorde a las necesidades salientes (Glenn, 2016; Serrano, 2016).

Así, como lo expresan Chumaceiro, Hernández y Chirinos (2016) "el nuevo escenario mundial de globalización ha hecho que las organizaciones se transformen, adapten y desempeñen nuevos roles" (p. 54). Por tanto los gobiernos y las organizaciones internacionales se han propuesto generar estrategias para contrarrestar los impactos negativos, como el Proyecto Milenio, creado con el objetivo de discutir y evaluar los futuros desafíos que puede tener la humanidad con las tecnologías 4.0 (Cordeiro, 2013; Fundación telefónica, 2018; Glenn, 2016).

\section{Tecnologías 4.0}

La evolución de la industria presenta tres (3) principales etapas: I) La llegada de la máquina de vapor, significando el cambio de un trabajo manual a uno mecanizado, II) la producción en masa, potencializada por Henry Ford y la producción en cadena entre los siglos XIX y XX, y III) la producción automatizada, con la expansión de los mercados y la globalización, producto de las Tecnologías de la Información y de las Comunicaciones-TIC (Oztemel \& Gursev, 2018). A lo anterior, llega la industria 4.0, asociada al internet y la sociedad del conocimiento y a las herramientas digitales que impulsan las nuevas formas de producción, flexibilizando la interacción entre lo digital y lo físico para aumentar la productividad. (Kagermann citado en Schneider, 2018; Villasmil y Crissien, 2015; Hernández y Chumaceiro, 2008).

En concordancia, Waldner (2008) identifica el término internet de las cosas, abordando la interconectividad de los objetos cotidianos al internet, y su digitalización, afectando así a las personas y a su forma de relacionarse con el entorno, e impactando también, a los empleados en sus tareas laborales (Kong, Luo, Huang \& Yang, 2018). De esta manera, la tecnología 4.0, supone un incentivo hacia la digitalización de forma integral y a la interconectividad entre lo físico y lo digital (Drath y Koziolek citados en Schneider, 2018). Como lo expresa Pérez (2019) "la innovación empresarial es una herramienta de gestión empresarial fundamental en el contexto de competitividad vigente" (p. 101).

Por su parte, Córdoba, Marcos, Aguayo \& De las Heras (2016) determinan que dentro de las tecnologías 4.0, se encuentran: 1) big data, 2) cloud computing, 3) fabricación e impresión $3 \mathrm{~d}, 4)$ robótica colaborativa y 5) sistemas ciberfísicos. Oztemel y Gursev (2018) suman las máquinas inteligentes o los robots, que actúan guiados por un programa informático preestablecido. Donde los robots, presentan un avance en su desarrollo y autonomía, solucionando problemas complejos de forma libre y aprendiendo a relacionarse con el entorno, generando un aumento de su presencia en las empresas (Rojas y Humberto, 2017).

Esto significa para un futuro cercano la existencia de un mayor fortalecimiento de la relación humanos-robots y en la presencia de robots humanoides (robots con aspecto humano) en las empresas. De esta forma, la robótica y los robots, serán la tendencia en ambientes riesgosos y en las tareas categorizadas como operarias y tradicionales (Ceballos, 2016). 
En complemento, la Inteligencia Artificial (IA), es la inteligencia de las maquinas o entidades digitales, desarrolladas para solucionar procesos complejos de forma eficiente, trabajar de forma colaborativa e inclusive aprender, emulando características de los humanos a partir de una programación base, generando conocimiento según su relación con su entorno (Lahoz-Beltrá, 2004; Oztemel y Gursev, 2018).

Ahora bien, Córdoba et al. (2016) y Pérez, Saucedo, Salais y Marmolejo (2016) identifican como ventajas de la industria 4.0., el aumento de la eficiencia en los procesos, mediante la potencialización de productos ya existentes a una interconectividad digital, flexible y personalizada, representando optimización de los recursos y agilidad para los clientes, mejor competitividad y asegurando la sostenibilidad. Además de ambientes de trabajo más seguros, entrenamiento personalizado, aumento de la autonomía, mayor enfoque por la innovación, monitoreo y control, menores costos de producción, incremento de la calidad de vida y mayor productividad (Kong et al., 2018).

Por ende, los retos están en la afectación a la privacidad, ataques cibernéticos, dificultad por mantener la propiedad intelectual, en la adaptación de las empresas a las nuevas tecnologías, en el desarrollo de procesos y estructuras flexibles, y en la incertidumbre sobre la desaparición de trabajos, reto que se puede afrontar desde la educación y la capacitación (Córdoba et al., 2016; Kong et al., 2018; Oztemel y Gursev, 2018).

\section{Tecnologías 4.0 y su incidencia}

Córdoba et al. (2016) exponen que los recursos más importantes en la industria 4.0 seguirán siendo el talento humano y la tecnología, por lo que las maquinas no van a sustituir al hombre, la diferencia radica en la mayor interconectividad, interacción, dependencia y colaboración (Nagamachi \& Lokman, 2015). Así, las empresas van a requerir mayores empleados con habilidades en innovación y en habilidades cibernéticas (Córdoba et al., 2016). No obstante, el cambio debe ser gradual, con el fin de facilitar el tránsito y la adaptación a las tecnologías (Schneider, 2018).

De igual manera, las tecnologías 4.0 descentralizaran la toma de decisiones, ampliando las capacitaciones, enfatizándolas en desarrollo de herramientas tecnológicas. Incrementando la productividad e impactando en la motivación, confianza y compromiso de los empleados, apostando por la autogestión, retroalimentación y autoevaluación (Porter \& Heppelmann 2015).

Por lo tanto, los requisitos y la clasificación del nuevo personal se enfocarán hacia la flexibilización y el análisis de los procesos automatizados, otra razón para que las tareas repetitivas sean ejecutadas por robots, potencializando el trabajo colaborativo entre las máquinas y los seres humanos, agilizando los procesos y fortaleciendo la trazabilidad. A su vez, implementando una comunicación virtual basada en dispositivos conectados a internet, táctiles e inteligentes, aumentando la eficiencia en las industrias (Córdoba et al., 2016; Kong et al., 2018; Schneider, 2018).

Al abordar los impactos económicos de la industria 4.0 en la sociedad, se pueden identificar dos tendencias. Por un lado, los detractores del actual ritmo de desarrollo sostienen que la concentración de la riqueza continuará en unos pocos, perpetuando la inequidad, siendo el crecimiento económico un producto de las nuevas tecnologías, más no, un impulso al empleo o a la distribución de ingresos (Fundación telefónica, 2018). 
En este sentido, Oztemel y Gursev (2018) identifican el reemplazo de la mano de obra no calificada por la robótica, donde estos avances serán pilar de los trabajos generando altas tasas de desempleo, aunque haya un grupo de productos que deben ser elaborados a baja escala por mano de obra tradicional permitiendo ello complementar la cadena de valor (Bonilla, Cardeño y Cardeño, 2015; Pérez, Hernández, Acosta y Chumaceiro, 2009). Sin embargo, argumentan como las necesidades sociales, culturales, políticas y económicas se convertirán en factores de cambio, implicando educación en desarrollos tecnológicos, programación, robótica e IA (Zheng et al., 2018).

Por otro lado, se estima que se abrirán nuevos empleos y mejor remunerados, así como un aumento de la innovación y del desarrollo tecnológico. Donde, los principales cambios serán en los países desarrollados, por su compromiso para automatizar las labores, y evitar fuertes fugas de cerebros a causa del aumento del desempleo (Serrano, 2016).

De esta forma, identificar el personal calificado y capacitado en conocimientos técnico-tecnológicos y de gestión humana, generará un aporte significativo para las empresas en la interrelación entre empleados y máquinas (Schneider, 2018). En este contexto, se espera que la mujer tenga una mayor participación laboral, eliminando las desigualdades de género a nivel económico y en los puestos gerenciales (Fundación telefónica, 2018).

No obstante, autores como Quaglia y Plata (2018) mediante el uso de prospectiva en su investigación para la Argentina, encuentran la ocurrencia de desplazamiento de puesto de trabajo tradicionales, operativos y de alto riesgo para la vida humana, así como la necesidad de realizar ajustes en los nuevos trabajos.
Igualmente, Serrano (2016), mediante el resultado de la encuesta Delphi global acentúa la fuerte crisis laboral que se presentará. Considerando que el $25 \%$ de la población mundial se encontrará desempleada en el 2050, especialmente las personas de la tercera edad. En contraste, el desempleo será característico de los países más ricos, y los países en vías de desarrollo serán los que cuenten con nuevas plazas de empleo.

Adicionalmente Hernández y Chumaceiro (2008), Glenn y Gordon (2000) y Kellner (2000), resaltan la importancia de la educación y de la capacitación con calidad para la población del futuro. Impactando dentro del mercado laboral e influyendo en la participación de las personas en todos los sectores económicos. De tal forma, se debe fundamentar en el mejoramiento de la educación a través del uso de las tecnologías 3.0 y 4.0, y la optimización de los entornos multimedia.

En concordancia, para el caso colombiano la literatura referente al estudio prospectivo en el desarrollo del Millennium Project es escasa. Por ende, en el estudio realizado por Mancipe y Pardo (2017), se resalta la importancia de las organizaciones y su impacto económico, social, político y medioambiental (Chumaceiro, Hernández y Chirinos, 2016), mediante la implementación de los nuevos desarrollos tecnológicos a los procesos y actividades, que buscan ajustarse a las nuevas demandas y el cumplimiento del objeto social de dichas instituciones.

En sí, la prospectiva se puede considerar como una herramienta de estudio sobre el futuro, en el cual se incluyen la construcción de escenarios y su elección (Kalil, 2016). Comprendiéndolo como los planteamientos estratégicos sobre escenarios proyectados, analizando los acontecimientos 
del pasado, el presente y su impacto sobre el futuro. Dicho análisis, es específico de lo que comúnmente se conoce como hechos portadores de futuro estratégico (Amaya, 2005; Buitrago, 2007; Cuervo y Guerrero, 2018; Godet y Durance, 2007; Mojica, 2006; Ortiz, 2013; Rey, Osorio \& Botero, 2009; Rico, 2016).

Con la premisa anterior, se presenta que, dentro del enfoque de esta investigación (Análisis prospectivista referencia del Proyecto del Milenio), se destaca la creciente incertidumbre por el impacto que va a tener el desarrollo tecnológico como respuesta a los cambios generados por la innovación, sobre la calidad y las oportunidades laborales para el año 2050, se incluye la identificación de los diferentes hechos portadores del futuro estratégico en Colombia, partiendo del ejemplo de los estudios realizados en países latinoamericanos, Europeos incluyendo los Estados Unidos (Fundación telefónica, 2018; Glenn y Gordon, 2000; Quaglia y Plata, 2018; Serrano, 2016).

\section{Métodos y Materiales}

\section{Método}

Se desarrolló una investigación de corte cualitativo, basada en un análisis prospectivo, utilizado en diferentes estudios para analizar los posibles escenarios del futuro y con esto dar respuesta a la pregunta de investigación (Mojica, 2008).

Dentro del desarrollo de la prospectiva se incluye el método Delphi, cuyo propósito es instruir a las organizaciones y países en la definición y escogencia de escenarios teniendo en cuenta la incertidumbre consecuente (Baena, 2015; Gándara y Osorio, 2016; Zartha et al, 2017; Godet y Durance, 2007; López, 2018).
Adicionalmente, al análisis prospectivo se adhiere el análisis morfológico desarrollado por Fritz Zwicky, el cual se fundamenta en la construcción de escenarios futuros correlacionados a los factores estratégicos, teniendo en cuenta una cantidad óptima de combinaciones de escenarios y factores estratégicos, para hacer efectivas las proyecciones (Mancipe y Pardo, 2017; Ritchey, 1998).

Finalmente, se tienen en cuenta la cruz de escenarios de Peter Schwartz, que se encuentra directamente relacionada con el análisis morfológico dentro del panorama prospectivo. Los ejes de Schwartz permiten la creación de coyunturas más tangibles, estableciendo las características del entorno y su comportamiento, llevando a la selección de los escenarios mejor estructurados y más pertinentes, con los cuales es posible llegar al futuro esperado (Peláez, Álvarez, Palacio, y Mazo, 2017).

\section{Materiales}

Se realizó un desarrollo conceptual acerca de las oportunidades y falencias que puede tener el país para el 2050, mediante un panel de discusión referente al Estudio Prospectivo de Tecnología, Trabajo y Educación desarrollado en el año 2018, en el cual participaron catedráticos especializados en el análisis del futuro laboral y tecnológico, mediante el uso de la prospectiva, entre los que estaban el Vicerrector de la Universidad Nacional de Colombia (Sede Bogotá), docentes investigadores de la Universidad Nacional (Colombia) y la Universidad Central (Colombia), y el asesor en prospectiva del Ejercito Nacional.

Durante el foro, se abordó el desarrollo de las nuevas tecnologías y herramientas que están y transformarán la vida y el trabajo en torno a la cuarta revolución 
industrial (González, 2017). Además, se abordó la preocupación por el futuro de la educación y los nuevos conocimientos que deben ser desarrollados en pro de las nuevas labores que se crearán, para proveer al capital humano de herramientas para desenvolverse dentro de los nuevos mercados laborales (Glenn, 2016; Glenn y Gordon, 2001; Ortiz, 2013; Quaglia y Plata, 2018; Serrano, 2016).

Adicionalmente, buscando identificar los puntos de convergencia entre los temas tratados en el foro, se realizó una encuesta virtual respecto al desarrollo tecnológico de Colombia para el 2050 dirigida a 198 estudiantes con nivel educativo de pregrado, especialización, maestría, doctorado, posdoctorado, vinculados a la Universidad Central en Bogotá (Colombia), teniendo en cuenta que será la población que se verá afectada con los procesos de cambio tecnológico y desarrollo de nuevas dinámicas de trabajo.

\section{Resultados}

\section{Escenario tecnológico para Colombia a 2050}

En este apartado se presentan, los puntos de estudio de convergencia entre la exposición de los expertos y los resultados de las encuestas de acuerdo con las metodologías de análisis prospectivo seleccionadas.

\section{Aplicación de encuesta a grupos de interés}

Se realizó una encuesta enfocada a población que tiene un nivel educativo superior, donde la mayor participación fue de los hombres $(60,61 \%)$ con nivel educativo de pregrado (19,70\%), especialización $(16,16 \%)$ y maestría $(12,63 \%)$.
Inicialmente, para el primer grupo de preguntas referentes a la fuerza de trabajo se presenta que, el $72,22 \%$ de la población entrevistada considera que la mayoría de las labores operacionales, tradicionales o manuales y las que implican un alto riesgo para la vida humana, pertenecientes a todos los rubros de la economía serán automatizadas en el año 2050. En este sentido, el $64,14 \%$ considera que el pueblo colombiano si está preparado para asumir los cambios tecnológico, teniendo presente que tan solo el 58,08\% infiere que se presentará una mejora en las plazas de empleo, estando directamente relacionado con una mejora salarial.

Seguidamente en las preguntas referentes a sociedad y cultura, se refleja que la muestra converge a inferir que la implementación de nuevas tecnologías de digitalización, inteligencia artificial y robótica, transformará la cotidianidad de la comunicación y la interacción en las áreas laborales para el 2050 (94,44\%).

Referente a la dinámica que puede tener la industria y el mercado laboral, se presenta que el despliegue y adopción de las nuevas tecnologías repercutirá sobre la forma en que se hacen las transacciones de compra y venta de todos los sectores económicos (16,67\% y $15,66 \%$ - 2050, respectivamente). La industria del futuro estará preparada para el desarrollo de nuevas plazas de empleo implicando una automatización de todas las labores pertenecientes a entornos predecibles $u$ operativos.

El gobierno es un actor trascendental, debido a que este puede influenciar en el desarrollo de las nuevas tecnologías partícipes de la revolución industrial 4.0. Por tanto, el $21,72 \%$ de la población entrevistada concuerdan en que las políticas de inversión en innovación y ciencia deben ser la prioridad para el 2050 (18,69\%). De 
igual forma, se debe incluir una transformación significativa sobre la administración de la gobernanza (20,71\%), que debe estar acorde a los objetivos sociales, de crecimiento económico y de reducción del rezago presente respecto a los demás países de la región.

Como se ha destacado, la educación es una variable determinante para el desarrollo de los países. En este sentido, el $13,13 \%$ y $11,62 \%$ de los encuestados concuerdan en cambiar las metodologías de enseñanza y aprendizaje en todos los niveles educativos, sobre todo, en la educación superior que debe estar sujeta a las transformaciones más trascendentales, adaptándose a las inmersiones de la inteligencia artificial, y la robotización del conocimiento para el 2050. Adicionalmente, consideran que gran parte de las profesiones actuales serán obsoletas en el futuro (10,61\%), siendo una prioridad el desarrollo de nuevos centros de estudios para cubrir la nueva demanda laboral.

Finalmente, se encuentra la educación como motor de los desarrollos tecnológicos. Presentándose que el $32,32 \%$ de la muestra alude que para el 2050, tanto las políticas de gobierno como las instituciones educativas, deben generar un desarrollo del sector investigativo. Logrando enfrentar así los retos que implica la cuarta revolución industrial, aumentando la competitividad del país y su participación en el mercado internacional, comandado por la inteligencia artificial, la robótica y la digitalización $(26,77 \%)$.

\section{Aplicación Método Delphi}

En concordancia, los anteriores resultados confluyen con las interpretaciones de los panelistas, partiendo de las dinámicas sociales y políticas de los últimos 5 años y, sobre todo, el entorno actual referente a la disminución del presupuesto nacional entre 2017 y 2018 en un 11\%, destinado a educación, desarrollo de innovación y tecnología (Anzola, 2017). Plantean una proyección negativa para Colombia en el 2050, debido a la contracción presupuestal que repercutirá en aumentar el rezago que tiene el país en desarrollo de investigación científica reflejada en innovación, tecnología y desarrollo de patentes, respecto a la región y los países desarrollados (Gómez y Mitchell, 2014; Naranjo y Calderón, 2010).

Adicionalmente, reflejándose esta condición en la precariedad de la calidad y el acceso al sistema educativo por parte de los jóvenes representado por la tasa de deserción de educación superior en el año 2016, la cual se encontraba en la variación del $46,1 \%, 56,9 \%$ y $52,1 \%$ para las categorías universitarias, técnicas y tecnológicas, respectivamente (Melo, Ramos, y Hernández, 2014). En este sentido, se presenta que en el mercado laboral bajo las condiciones macroeconómicas y legales del país no logrará absorber toda la oferta de mano de obra, siendo referencia el aumento de la tasa de desempleo de 9,2\% en el 2016 al 9,4\% en el 2017 (República de Colombia. Banco de la República, 2018).

\section{Análisis Morfológico}

El desarrollo del análisis morfológico se realiza a través del planteamiento de una matriz de escenarios desplegados mediante el método Delphi, de cada variable estudiada. Por lo tanto, en la tabla 1 se muestran los escenarios negativos y positivos, de acuerdo con los resultados interpretados en el apartado anterior, que se pueden presentar en el país durante el desarrollo de la cuarta revolución industrial en el año 2050. 
TABLA 1.

Matriz de escenarios morfológico.

\begin{tabular}{|c|c|c|}
\hline Variable & Escenario Positivo & Escenario Negativo \\
\hline $\begin{array}{l}\text { Cuarta } \\
\text { Revolución } \\
\text { Industrial } \\
- \\
\text { Tecnología }\end{array}$ & $\begin{array}{l}\text { Colombia se adapta a la transformación tecnológica } \\
\text { e innovativa presente en el } 2050 \text {. Participación del } \\
64,14 \% \text { de la encuesta. }\end{array}$ & $\begin{array}{l}\text { El desarrollo tecnológico es limitado, } \\
\text { condicionando el acceso a la población. Lo } \\
\text { que no permite una adaptabilidad acorde } \\
\text { a la tendencia mundial. Profundizado por } \\
\text { un modelo económico no sostenible y poco } \\
\text { compatible con el medio ambiente. } 35,86 \% \text { de } \\
\text { la población encuestada. }\end{array}$ \\
\hline Educación & $\begin{array}{l}\text { Los centros educativos se especializan en } \\
\text { la generación, gestión y virtualización del } \\
\text { conocimiento. Significando el 13,13\% de la muestra. }\end{array}$ & $\begin{array}{l}\text { Por la poca disposición de recursos para } \\
\text { innovación en el sector educativo. Colombia } \\
\text { se rezaga en el desarrollo de tecnologías, } \\
\text { respecto a los países de la región y los } \\
\text { desarrollados. Representando el 18,69\% de } \\
\text { los estudiantes entrevistados. }\end{array}$ \\
\hline Trabajo & $\begin{array}{l}\text { Se presenta un escenario, en el cual las empresas } \\
\text { procuran absorber la mayor oferta laboral de las } \\
\text { nuevas profesiones que se crearán para el } 2050 . \\
\text { Permitiendo un control de las tasas de desempleo. } \\
\text { Proyectando el } 16,67 \% \text { de la participación } \\
\text { considerada como positiva. }\end{array}$ & $\begin{array}{l}\text { Se presentan altas tasas de desempleo, } \\
\text { profundizando los índices de pobreza y } \\
\text { subdesarrollo del país. Siendo reflejo de la } \\
\text { dinámica actual de la pésima vinculación } \\
\text { laboral (9,4\% año 2017). }\end{array}$ \\
\hline
\end{tabular}

Fuente: Elaboración propia con base en encuestas aplicadas y foro.

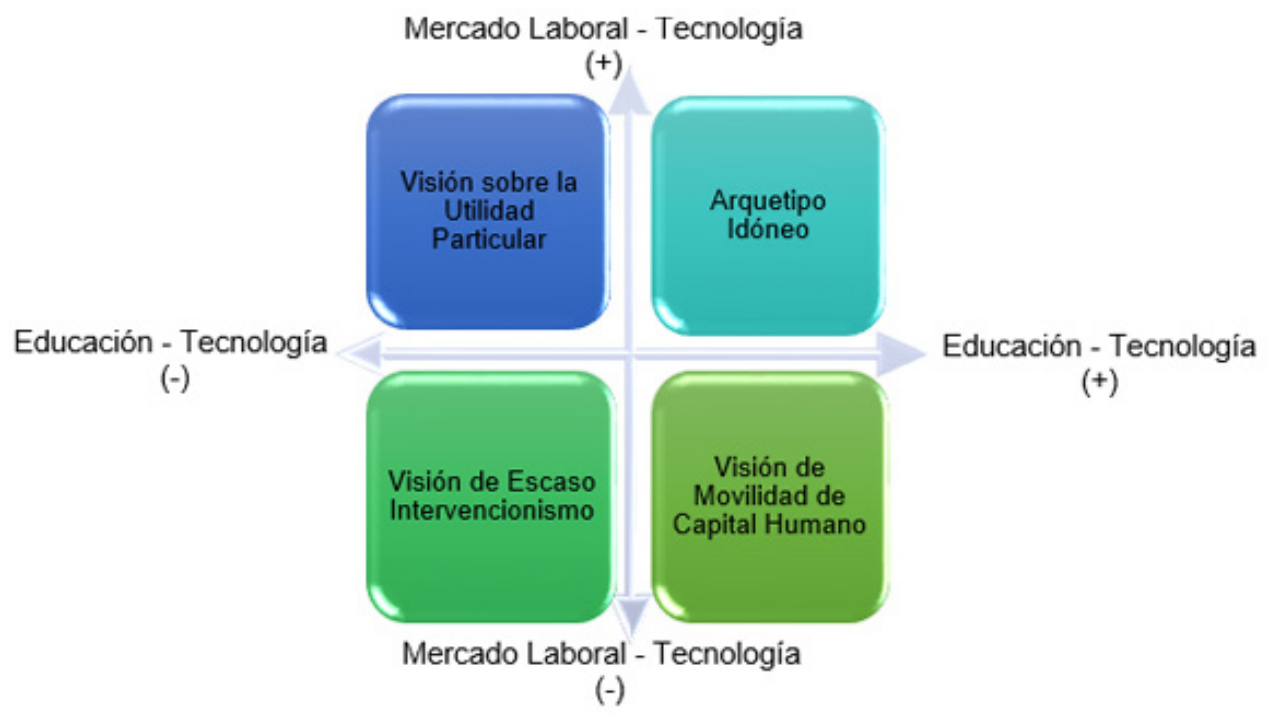

Figura 1. Cruz de escenarios de Peter Schwartz.

Fuente: Elaboración propia.

\section{Cruz de escenarios de Peter Schwartz}

La cruz de escenarios de Peter Schwartz busca plantear marcos o contextos más tangibles acerca del futuro, que implican una mayor probabilidad de ocurrencia y a los cuales se les puede dar una apuesta desde el presente (Peláez, Álvarez, Palacio y Mazo, 2017). Por lo tanto, a partir de los escenarios desarrollados en los apartados anteriores, respecto a nuestro documento los ejes de Schwartz se estructuran en la Figura 1. 
En dónde, la intersección de los vértices permite plantear las relaciones presentes entre la Educación y el mercado laboral con la tecnología, respectivamente. A su vez, de plantear la proyección del largo plazo para Colombia, con los posibles escenarios y medidas que puedan intentar proveer una visión positiva del 2050 .

\section{Arquetipo Idóneo}

En el primer cuadrante, se presenta el escenario idóneo o esperado para el 2050. En el convergen puntos fundamentales del desarrollo educativo y empresarial, que crean un contexto de ganancia mutua y crecimiento sostenido en el tiempo (fundamentado en la encuesta y el foro). Así, estos dos actores se enfocan en una constante generación de oferta y demanda de innovación y tecnologías, que son provistas inicialmente por las instituciones educativas, que suministran el conocimiento inicial al capital humano.

Seguido, de la creación de nuevos avances y conocimientos desarrollados en los conglomerados empresariales, que soportan el mercado laboral y logran absorben en su mayoría la sobre oferta de mano de obra capacitada. En este contexto de ganancia social y económica, juega un papel relevante la regulación gubernamental, al proveer de recursos financieros a las instituciones educativas para desarrollar investigaciones que permitan generar innovaciones acordes a los objetivos sociales y medioambientales, y regulando el comportamiento de las empresas frente a las tasas de desempleo.

\section{Visión sobre la Utilidad Particular}

Identificado en el segundo cuadrante, es un escenario en el cual la relación de la educación respecto a la tecnología es negativa, y la existente entre el mercado laboral y la tecnología o desarrollo innovativo en positiva.
Infiriendo de este escenario, se plantea poca preocupación del gobierno (inversión), sumado a la escasez de recursos financieros en los claustros educativos para la generación de nuevo conocimiento (escasez de recursos financieros), conlleve a una sobre oferta de mano de obra no calificada, aumentando las tasas de desempleo. De tal forma, que las empresas, inicien a demandar mano de obra extranjera y deteriorando las condiciones de las plazas de empleo disponibles, para aquellos que logran acceder a estas.

\section{Visión de Escaso Intervencionismo}

El tercer cuadrante, refleja el peor escenario que se puede esperar para el año 2050, reflejando que no se realice ningún tipo de cambio estructural tanto en la legislación como en el ámbito social. Principalmente, por la ineficiencia y la limitación de los esfuerzos de las empresas para crear las plazas de trabajo suficientes a las nuevas labores que se desarrollarán en este futuro.

A su vez, del rezago que se presentará en las universidades y demás claustros educativos, que procuran estar acorde con la tendencia mundial de conocimiento y de capacitación de la población. Sumado, a la poca convergencia de los objetivos conjuntos de los sectores empresariales y las instituciones educativas, que generaran una fuerte fuga de cerebros, al no proveerse de un escenario positivo para la proyección personal y profesional de las personas.

\section{Visión de Movilidad de Capital Humano}

En este último escenario, se presenta que la educación respecto a la tecnología tiende a tener un comportamiento positivo, reflejado en el creciente desarrollo innovativo y el aumento del capital humano capacitado que se traduce en una sobre oferta laboral. La cual, no puede ser completamente absorbida por 
el mercado empresarial, derivando en una contracción del crecimiento económico.

Por consiguiente, el escenario proyectado muestra un entorno negativo para los nuevos profesionales que no logran alcanzar un puesto laboral acorde a sus capacidades, conocimientos y habilidades adquiridas desde la investigación en las instituciones educativas. Creando un contexto de fuerte migración, a plazas de mercado extranjeras que logren aprovechar los conocimientos enfocados hacia la innovación, presentando una desventaja competitiva respecto a los demás países de la región.

\section{Propuesta de hechos portadores de futuro estratégico - Colombia 2050}

En complemento al desarrollo metodológico y para el posterior planteamiento del escenario de apuesta posibles para Colombia en el año 2050 , se tiene en cuenta que los encuestados, hicieron referencia a las posibilidades de adaptabilidad que puede tener la población en el proceso de transformación tecnológica, en donde el 19,19\% y el 17,68\% de personas con un nivel académico de pregrado y especialización, respectivamente, consideran que la población en general apoyará el desarrollo tecnológico, teniendo presente que resistirse al cambio solo generará un impacto negativo sobre la población más vulnerable.

En este mismo sentido, la muestra en todos los niveles educativos considera que las carreras que seguirán vigentes en el 2050 serán las enfocadas en Sistemas e Informática y todas las ingenierías. Visualizando un panorama poco alentador para las áreas de artes, filosofía y letras, logística, derecho y ciencias económicas y contables, que deberán tener una nueva adecuación para el futuro automatizado y robotizado, que reemplazará la mayoría de las labores que estas carreras desempeñan actualmente.
Confluyendo con las conclusiones de los participantes en el foro, proponen una serie de hechos portadores de futuro estratégico, que pueden ayudar a mejorar la expectativa del futuro educativo y laboral, incluyendo el desarrollo innovativo:

a. El sistema educativo, siendo el principal factor de transformación de la economía y la sociedad, debe tener un cambio enfocado en la generación y gestión de conocimiento, de tal forma que se reduzca la brecha innovativa y tecnológica que se tiene respecto a los países desarrollados.

b. Las universidades deben hacer un cambio de pensum, con el cual se logre el desarrollo de habilidades de percepción, intuición, comunicación, identificación y creación de patrones de los estudiantes, implicando un cambio pedagógico de transmisión del conocimiento a través de su virtualización.

c. En el país se debe desarrollar un nuevo escenario de congruencia entre el sistema educativo y el sector real, teniendo como objetivo la capacitación para el trabajo y crecimiento exponencial de las empresas, de tal forma que estas provean de los espacios laborales adecuados para la explotación del conocimiento adquirido.

d. El gobierno debe generar un cambio en los procesos de explotación sostenible de los diferentes commodities del país. Garantizando la estabilidad económica suficiente para incentivar nuevamente la migración de la población campesina de las ciudades a las zonas rurales. Diversificando los bienes que pueden ser referente de exportación y disminuyendo la participación del petróleo como el principal commodity de la economía colombiana.

En consecuencia, con estos planteamientos, a pesar de que algunas personas consideran que puede existir una proyección 
positiva de lo que puede suceder en el futuro para Colombia, siendo planteado sobre un escenario ideal de mejoramiento de las condiciones educativas y laborales para el país. Realmente, la visualización de acuerdo con la realidad actual refleja un contexto pesimista, en la medida que, para cumplir con las expectativas del futuro y poder estar a la par tanto con los países desarrollados como los de la región, y poder recibir los cambios esperados de innovación y nuevas tecnologías, se debe:

a. Plantear un reto conjunto entre la academia, el sector empresarial y el gobierno, en el cual, se disminuyan las brechas de analfabetismo, de pobreza y de acceso a la educación acorde a las nuevas necesidades del mercado para toda la población nacional (Clavijo, 2010).

b. Procurar incentivar el desarrollo de nuevas empresas que permitan absorber la sobre oferta de mano de obra disponible.

c. Disminuir la corrupción y la malversación de los fondos públicos, que hasta el momento no han permitido el óptimo progreso del país, teniendo en cuenta las ventajas competitivas de producción y explotación de recursos naturales frente al mercado global (Mancipe y Pardo, 2017).

\section{Conclusiones y RECOMENDACIONES}

Como bien se analizó en el apartado anterior, la proyección que se tiene para Colombia tiende a ser levemente positiva, tanto en los resultados de la encuesta como los desarrollados en el foro; siempre y cuando se realicen los cambios económicos, sociales, políticos y, sobre todo, culturales necesarios, para disminuir la probabilidad de tener un escenario negativo. Para lo cual, se deben seguir los siguientes puntos:

\section{Cambios sociales}

La sociedad, es el pilar de los cambios más significativos y trascendentales, en dónde:

4. La sociedad debe crear una conciencia generalizada desde el presente, de los fuertes cambios que presentará el futuro y afectarán la vida cotidiana.

5. A su vez, debe haber un desarrollo intelectual y académico autónomo de las personas, haciendo uso de las herramientas tecnológicas.

\section{Cambios Económicos}

Enfocado en los ámbitos laborales y financieros, especificando:

1. Creación de un objetivo específico al cual se quiera llegar en el 2050, incluyendo al sector gobierno, empresarial y laboral.

2. Desarrollo de un compromiso por parte de los empresarios ante el gobierno y la sociedad, de permitir la mayor vinculación laboral posible tanto de mano de obra capacitada como en proceso de capacitación y no capacitada, buscando disminuir las brechas de pobreza y las tasas de desempleo (García, 2018).

3. Reestructuración económica, pasando de depender de un commodity a diversificar la oferta de productos que se pueden exportar y potencializar su consumo nacional e internacional.

4. Es necesario que la población migrante a las ciudades regrese al campo, potencializando su explotación sostenible y asegurando un futuro para esta población

5. La automatización, la robótica y la inteligencia artificial, se consideran los factores que producirá mayor deserción tanto laboral como universitaria. Por tal, es ne- 
cesario una mayor disposición de recursos para las instituciones educativas, especializadas en investigación y desarrollo de innovación.

6. La transformación digital, como un proceso previo a la implementación tecnológica empresarial y las oportunidades que este proceso conlleva.

\section{Cambios políticos}

La política y la gobernabilidad, siendo el actor que debe transformarse en pro del bienestar colectivo, se sugiere:

1. Generar las regulaciones necesarias para contrarrestar la corrupción que siempre ha afectado a Colombia a lo largo de su historia. En la medida que, los fondos públicos que se pierden puedan ser redistribuidos en todos los rubros que presentan falencias dentro de la estructura económica como: el sistema educativo, el sistema de salud, infraestructura vial a nivel nacional, etc.

2. Desarrollo de leyes conjuntas entre la población y el gobierno, para proteger los recursos naturales y las áreas categorizadas como patrimonio natural del país.

\section{Cambios culturales}

Considerándose un factor difícil de modificar, es necesario que se realicen cambios a las costumbres de la población, para lograr su adaptabilidad a la cuarta revolución industrial. Con lo cual se debe contar con un trabajo gubernamental riguroso, que implique el acercamiento a las comunidades más aisladas de las urbes y capacitar a esta población, en pro del mejoramiento territorial y de las condiciones de calidad de vida que incluye el acceso a la educación, salud, infraestructura de alcantarillado y la disponibilidad de vinculación laboral.
En consecuencia, este documento genera un nuevo espectro de futuras investigaciones implicando analizar específicamente, la proposición de herramientas para las universidades y demás instituciones educativas, incrementando sus índices de investigación y desarrollo de innovación, mejorando la capacitación de los futuros profesionales. Igualmente, desarrollar estrategias de mejoramiento social, incentivando a las personas a capacitarse y posteriormente generando emprendimiento, que implique la disminución de las tasas de desempleo.

\section{REFERENCIAS}

Amaya, J. (2005). Gerencia: planeación \& estrategia. Bogotá, D.C.: Universidad Santo Tomas de Aquino.

Anzola, G. (2017). Financiación o desfinanciación-inclusión o exclusión ¿Qué existe para la educación superior? Revista UDCA Actualidad \& Divulgación Científica, 20(2), 237-239. https://doi.org/10.31910/rudca. v20.n2.2017.378

Baena, G. (2015). Planeación Prospectiva Estratégica. Teorías, Metodologías y Buenas Prácticas en América Latina. [Proyecto Papime No. PE300414]. México D. F.: UNAM.

Bonilla, E., Cardeño, E. y Cardeño, N. (2015). La función financiera en las micros, pequeñas y medianas empresas, del municipio de Riohacha. Económicas CUC, 36(2), 137-146

Buitrago, C. (2007). La incertidumbre de futuro y la estrategia empresarial. Revista AD-MINISTER, (11), 97-131.

Caro, J. (2016). Financiación de la innovación tecnológica en el sector servicios en Colombia. Económicas CUC, 37(2), 89-114. http://dx.doi.org/10.17981/econcuc.37.2.2016.05

Castells, M. (1999). La revolución de la tecnología de la información. En, M. Castells, $L a$ era de la revolución: economía, sociedad y cultura, Bogotá, D.C.: Siglo XXI. 
Clavijo, G. (2010). La universidad colombiana de cara al 2050. Teoría y praxis investigativa, 5(1), 43-58.

Ceballos, V. (2016). Industria 4.0, la gran oportunidad. Economía Aragonesa, (59), 109-123.

Chumaceiro, A., Hernández, J. y Chirinos, E. (2016). Responsabilidad social universitaria, desarrollo sostenible y ciudadanía ambiental. Cuadernos de RSO, 4(1), 53-64. Disponible en https://ucu.edu.uy/ sites/default/files/facultad/fce/rso/cuadernos-de-rso-vol-4-n1-chumaceiro.pdf

Chumaceiro, A., Hernández, J., Meléndez, Y. y Acurero, M. (2017). Responsabilidad universitaria y sociedad del conocimiento para la generación de competencias TIC. En, A. Chumaceiro, F. Ramos y J. Hernández (Ed), Procesos formativos para el siglo XXI. Cabimas: Fondo editorial UNERMB.

Cordeiro, L. (2013). The Millennium Project and beyond. Foresight, 15(5). [Online]. https://doi.org/10.1108/FS-03-2013-0008

Córdoba, A., Marcos, M., Aguayo, F. \& De las Heras, A. (2016). State Of The Art Of Kansei Engineering For Projection In Industry 4.0. In, AEIPRO, 20th International Congress on Project Management and Engineering. Cartagena, Colombia. Disponible en https://www. aeipro.com/files/congresos/2016cartage na/03043.4613.pdf

Cruz, H. y Gutiérrez, R. (2018). Un estudio prospectivo del trabajo en Colombia al 2050. Innovación y Desarrollo Tecnológico, 10(4). 123-138.

Cuervo, L. y Guerrero, F. (eds.) (2018). Prospectiva en América Latina: aprendizajes a partir de la práctica. Seminarios y conferencias (88). Santiago de Chile: Cepal.

Fundación Telefónica. (abril 5, 2018). El trabajo y la revolución digital en España para 2050. [Online]. Recuperado de: https://www.fundaciontelefonica.com/ arte_cultura/publicaciones-listado/pagina-item-publicaciones/itempubli/636/
Gándara, G. y Osorio, F. (Coords.) (2016). La Prospectiva y sus métodos procedimentales para la anticipación del futuro. CONfines de Relaciones Internacionales y Ciencia Política, 12(22), 131-138. García, F. (2018). El Mercado Laboral y La Cuarta Revolución Industrial. Capital Humano, 31(330), 20-21. Recuperado de http://ezproxy.javeriana.edu.co:2048/ login?url=http://search.ebscohost.com/ login.aspx?direct $=$ true $\& \mathrm{db}=$ fua $\& \mathrm{AN}=1$ 29254696\&lang=es\&site=eds-live

Glenn, J. (2016). 2050 Global work/Technology scenarios. The Millennium Project Team. [Online]. Recuperado de: http://107.22.164.43/millennium/WorkTech-2050-Scenarios.pdf

Glenn, J. \& Gordon, T. (2001). The Millennium Project: Challenges We Face at the Millennium. Technological Forecasting and Social Change, 66(2-3), 129-312.

Glenn, J. \& Gordon, T. (2000). Views from the millennium project on the future of technology: On the threshold: The United Nations and global governance in the new millennium. In, United Nations University, On the Threshold: The United Nations and Global Governance in the New Millennium. Tokyo, Japan

Godet, M., y Durance, P. (2007). Prospectiva Estratégica: problemas y métodos. Cuadernos de LIPSOR, 104. París: DUNOD

Gómez, H. y Mitchell, D. (2014). Innovación y emprendimiento en Colombia-Balance, perspectivas y recomendaciones de política: 2014-2018. Cuadernos de Fedesarrollo (50). BOGOTÁ, D.C.: Fedesarrollo.

González, J. (2017). Cuarta Revolución Industrial, Empleo y Estado de Bienestar. Madrid: Real Academia de las Ciencias Morales y Políticas.

Hernández, J. y Chumaceiro, A. (2008). Sociedad del conocimiento y pertinencia social universitaria. CEISEP una propuesta para la UNERMB. Revista Venezolana de Ciencias Sociales, UNERMB, 12(1), 77-92. 
Kalil, J. (2016). Prospectiva para la investigación en la Facultad de Ciencias Económicas y Administrativas de la Universidad Católica de Santiago de Guayaquil [Tesis de Maestría]. Universidad Católica de Santiago de Guayaquil, Guayaquil, Ecuador.

Kellner, D. (2000). New technologies/ new literacies: Reconstructing education for the new millennium. Teaching Education, 11(3), 245-265. https://doi. org/10.1080/713698975

Kong, X. T., Luo, H., Huang, G. Q. \& Yang, X. (2018). Industrial wearable system: the human-centric empowering technology in Industry 4.0. Journal of Intelligent Manufacturing, 30(8), 2853-2869. https://doi.org/10.1007/s10845-018-14169

Lahoz-Beltrá, R. (2004). Bioinformática: simulación, vida artificial e inteligencia artificial. Madrid: Díaz de Santos.

López, E. (2018). El método Delphi en la investigación actual en educación: una revisión teórica y metodológica. Educación $X X 1,21(1), 17-40$.

Mancipe, F. y Pardo, I. (2017). Estudio Prospectivo de la Organización Acceso Colombia ESAL al año 2026. [Tesis de Maestría]. Universidad Externado de Colombia, Bogotá, D.C., Colombia.

Melo, L., Ramos, J. y Hernández, P. (2014). La educación superior en Colombia: situación actual y análisis de eficiencia. Borradores de Economía, (808). 1-50.

Mojica, F. (2008). Dos modelos de la escuela voluntarista de prospectiva estratégica. Bogotá, D.C.: Universidad Externado de Colombia.

Mojica, F. (2006). Introducción a la prospectiva estratégica. Bogotá, D.C.: Publicaciones Capal.

Nagamachi, M. \& Lokman, A. M. (2015). Kansei innovation: practical design applications for product and service development. Boca Ratón: CRC Press.
Naranjo, J. y Calderón, G. (2010). La investigación en innovación en Colombia y México. Un análisis desde la difusión en revistas científicas. Dyna, 77(162). 191203.

Ortiz, E. (2013). Análisis del entorno y prospectiva. Illes Balears: IMP.

Oztemel, E. \& Gursev, S. (2018). Literature review of Industry 4.0 and related technologies. Journal of Intelligent Manufacturing, 1-56. https://doi.org/10.1007/ s10845-018-1433-8

Peláez, M., Álvarez, Y., Palacio, I. y Mazo, A. (2017). Aplicación de los ejes de Schwartz como metodología de prospectiva tecnológica al modelo universitario-empresa en el contexto colombiano. Revista Ingenierías USBMed, 8(1), 63-70. https://doi. org/10.21500/20275846.2772

Pérez, C. (2019). Innovación empresarial al servicio de la micro y pequeña empresa nortesantandereana: por la competitividad regional. Económicas CUC, 40(1). 91-104. http://doi.org/10.17981/econcuc.40.1.2019.06

Pérez, M. E., Hernández, J., Acosta, I. y Chumaceiro, A. (2009). Consideraciones teóricas para el análisis de las Pequeñas y Medianas Empresas como fuente de generación de empleo y su correspondencia ética con la Sociedad. Revista Formación Gerencial, 8(2). 272-297

Pérez, M., Saucedo, J., Salais, T. y Marmolejo, J. (2016). Caracterización de modelo de negocio en el marco de industria 4.0. En, Congreso Internacional de Logística y cadena de suministro, CiLOG2016. Mérida, México.

Porter, M. E. \& Heppelmann, J. E. (2015). How smart, connected products are transforming companies. Harvard Business Review, 93(10), 96-114.

Quaglia, N. y Plata, B. (2018). Propuesta de Proyecto de Investigación: "Argentina 2050: El futuro de la tecnología y el trabajo”. [Proyecto de investigación UCES]. Buenos Aires: CeLGyP. 
República de Colombia. Banco de la República. (2018). Boletín de Indicadores Económicos. [Online]. Recuperado de http:// www.banrep.gov.co/sites/default/files/paginas/bie.pdf

Rey, R., Osorio, J. y Botero, C. (2009). Factores de éxito y hechos portadores de futuro para consolidar un observatorio para la competitividad en Risaralda. Scientia et technica, 15(41), 165-170.

Rico, S. (2016). La investigación para la construcción de escenarios ante la toma de decisiones. En, UNLP, VIII Congreso de Relaciones Internacionales, La Plata, Argentina.

Ritchey, T. (1998). General morphological analysis. In, 16th euro conference on operational analysis. Brussels, Belgium.

Rojas, C. y Humberto, J. (2017). La Cuarta Revolución Industrial o Industria $4.0 \mathrm{y}$ su Impacto en la Educación Superior en Ingeniería en Latinoamérica y el Caribe. 15th LACCEI International MultiConference for Engineering, Education, and Technology: "Global Partnerships for Development and Engineering Education”, Boca Raton Fl, United States.

Schneider, P. (2018). Managerial challenges of Industry 4.0: an empirically backed research agenda for a nascent field. Review of Managerial Science, 12(3), 803-848. http://doi.org/10.1007/s11846-018-0283-2

Serrano, R. (2016). El futuro del trabajo y la tecnología: Investigación prospectiva en el marco del proyecto Millenium. Hermes: pentsamendu eta historia aldizkaria, (53), 60-66.

Villasmil, M. y Crissien, T. (2015). Cambio de paradigma en la gestión universitaria basado en la teoría y praxis de la reingeniería, Económicas CUC, 36(1), 245-274. http://dx.doi.org/10.17981/econcuc.36.1.2015.29

Waldner, J.-B. (2018). Nanocomputers and swarm intelligence. Hoboken: John Wiley \& Sons. http://dx.doi. org/10.1002/9780470610978
Zartha, J., Montes, J., Toro, I., Hernández, R., Villada, H. y Hoyos, J. (2017). Método Delphi en estudios de prospectiva tecnológica: una aproximación para calcular el número de expertos y aplicación del coeficiente de competencia experta 'k'. Biotecnología en el Sector Agropecuario y Agroindustrial, 15(1), 105-115. Zheng, P., Sang, Z., Zhong, R. Y., Liu, Y., Liu, C., Mubarok, K. \& Xu, X. (2018). Smart manufacturing systems for Industry 4.0: Conceptual framework, scenarios, and future perspectives. Frontiers of Mechanical Engineering, 13(2), 137-150. https://doi.org/10.1007/s11465018-0499-5

\section{Biodata}

Liliana Carolina Rodríguez Valero es Administradora de Empresas de la Universidad Nacional Abierta y a Distancia (Colombia). Candidata a la Maestría en Gestión de Organizaciones de la Universidad Central (Colombia). Funcionaría del Departamento de Publicidad de la Universidad Central. https://orcid.org/0000-00019799-6729

\section{Ramón Eduardo Gutiérrez Rodríguez} es Administrador de Empresas de la Universidad Nacional (Colombia). Especialista en Evaluación Social de Proyectos de la Universidad de los Andes (Colombia). Magister en Investigación en Estudios Internacionales en Ciencias Sociales y Humanas de la Universidad de París 3 (Francia). Estudiante de Doctorado en Comunicación de la Universidad Nacional de la Plata (Argentina). Docente Tiempo Completo del Departamento de Administración de Empresas de la Universidad Central (Colombia). https:// orcid.org/0000-0001-9928-4936 